\title{
Malposition of a Nasogastric Tube: A Case Report
}

Rajeeka Nadeeshani* and Ranmali De Silva Kulasiri

Department of Anaesthesiology, National Hospital, University of Colombo, Sri Lanka

*Corresponding author: Rajeeka Nadeeshani, National Hospital, University of Colombo, Sri Lanka, Tel: + +94772245931; E-mail: rajee.nadeeshani@gmail.com

Received Date: March 08, 2018; Accepted Date: March 22, 2018; Published Date: March 28, 2018

Copyright: @2018 Nadeeshani R, et al. This is an open-access article distributed under the terms of the Creative Commons Attribution License, which permits unrestricted use, distribution, and reproduction in any medium, provided the original author and source are credited.

\begin{abstract}
Malposition of a nasogastric tube in a 65-year-old patient with a laceration in the oesophagus following an accidental ingestion of a denture is presented in this report. Malposition of a nasogastric tube would mostly lead to unexpected fatal complications unless the placement is confirmed accurately. In the presented case however, fatal pulmonary complications were successfully prevented through timely detection and confirmation by the chest X-ray.
\end{abstract}

Keywords: Malposition of nasogastric tube; Air insufflation; Epigastric auscultation; $\mathrm{pH}$ testing; Chest X-ray

\section{Introduction}

Nasogastric tube insertion is a very common procedure often performed in intensive care settings, emergency departments or hospital wards, whenever access to the stomach is needed for diagnostic and therapeutic procedures. Therapeutic indications for the insertion includes gastric decompression, aspiration of gastric contents, feeding and administration of medication.

\section{Case Presentation}

A 65 year old lady, diagnosed with hypertension was admitted to the surgical ward following an accidental ingestion of her denture. She had a BMI of $32 \mathrm{~kg} / \mathrm{m}^{2}$, pulse rate of $70 \mathrm{bpm}$ and blood pressure of $110 / 70$ mmHg, saturation of $96 \%$ on air. A rigid oesophagoscopy was performed under general anaesthesia with rapid sequence induction. The denture was visualized at $17 \mathrm{~cm}$ distance from the incisor teeth. Oesophagoscopy reveled a laceration in the posterior wall at $15-17 \mathrm{~cm}$ from the upper incisor teeth. The removal was attempted twice but failed. An iotrogenic laceration was noted on the posterior wall of the oesophagus at $31 \mathrm{~cm}$ from the incisor teeth. Surgery was abandoned and she was extubated once fully awake and transferred to the ward.

Patient was kept nil by mouth, an intravenous crystalloid $100 \mathrm{ml} / \mathrm{h}$ was given for the maintenance requirement. Contrast enhanced CT with oral contrast was performed on the same day. It revealed pneumo mediastinum with contrast leakage at the level of seventh cervical vertebra. A right sided basal lung collapse and consolidation was also noted.

The patient developed two episodes of haemoptysis. Subsequently she developed a tachypnea of 40 breaths per min, saturation dropped to $86 \%$ on air with reduction of air entry to the right lung. Rigid oesophagoscopy was done under general anaesthesia on the next day and postoperative intensive care arranged. The denture with two teeth and hard palate was removed. Two tears at the level of $17 \mathrm{~cm}$ and 31 $\mathrm{cm}$ was noted on the posterior and the lateral wall respectively which the thoracic surgeon planned to manage conservatively.

Following the completion of the surgery, a decision to insert a nasogastric tube under direct vision was made collectively by the ENT surgical team and the anaesthetic team for the purpose of feeding till a jejunostomy or gastrostomy is performed by the general surgical team. The nasogastric tube was inserted under the direct vision with the oesophagoscope. Successful placement of the nasogastric tube was confirmed by the air insufflation and epigastric auscultation, following which the patient was transferred to the surgical intensive care unit with the endotracheal tube and nasogastric tube in situ.

Upon stabilization, antero posterior chest X-ray was performed before the commencement of feeding by the nasogastric tube. The nasogastric tube was noted within the lung parenchyma. It was reported by the consultant radiologist who revealed that the naso gastric tube has probably come out from the laceration of the oesophagus and entered the pleura of the right lung and the tip placed at the right base (Figure 1).
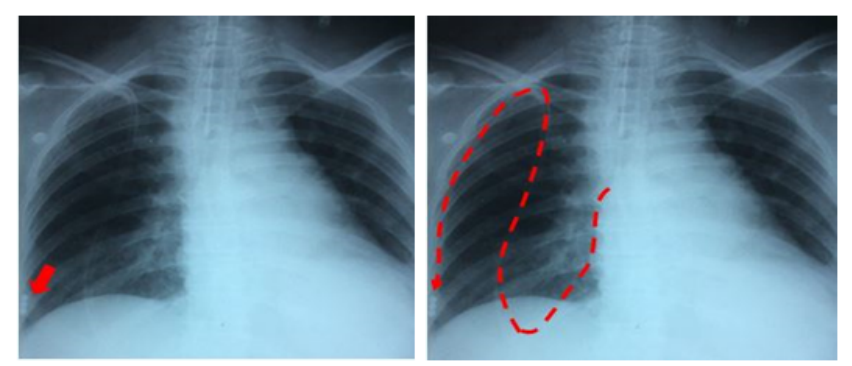

Figure 1: ENT surgical team was immediately informed and the nasogastric tube was gently pulled out.

\section{Discussion}

Insertion of the nasogastric tube is considered as a simple, easy and most uneventful procedure. The rate of malposition of the naso gastric tubes to the trachea and the distal airways were reported as $2-2.5 \%$. Sorokin and Gottlieb, Pennsylvania USA reported 50 cases of malposition of nasogastric tube to the right or left main bronchus out of 2000 nasogastric tube insertions over a period of four years which was complicated with two mortalities [1].

Malposition of the nasogastric tube causes complications such as pneumothorax, pleural effusion, retro pharyngeal abscess and lung 
abscess and even death. Therefor the placement of the tip of the nasogastric tube should be confirmed before commencement of feeding.

Though the air insufflation and epigastric auscultation are most commonly used method of confirmation, good thoraco abdominal sound transmission may cause misinterpretation even with tracheo bronchial misplacement of nasogastric tube [2].

According to National Patient Safety Agency 2011 (NPSA)-NHS guidelines, the placement of the tip is confirmed by,

- First line test method-pH testing with the $\mathrm{pH}$ range of 1-5.5

- Second line test method-chest X-ray when no aspirate could be obtained or $\mathrm{pH}$ indicator paper has failed to confirm the location.

The $\mathrm{pH}$ between 1-5.5 excludes the pulmonary placement but does not confirm the gastric placement as there is a small possibility of sitting the tip of the tube at the distal oesophagus which carries a high chance of aspiration [3].

Therefore chest X-ray must be performed to confirm the placement before feeding.

\section{Conclusion}

Nasogastric tube insertion although considered as an easy procedure can lead to unexpected complications as it is in this case report. Thus placement of the nasogastric tube must be confirmed in more than one method as we did which prevented a major morbidity.

\section{Declaration of Interest}

None

\section{References}

1. Sorokin R, Gottlib J (2006) Enhancing patient safety during feeding-tube insertion. A review of more than 2000 insertions. JPEN J Parenter Enteral Nutr 30: 440-445

2. Raut MS, Joshi S, Maheshwari A (2015) Malposition of a nasogastric tube. Ann Card Anaesth 18: 272-273

3. Anonymous (2011) Patient Safety Alert NPSA/2011/PSA002: Reducing the harm caused by misplaced nasograstric feeding tubes in adults, children and infants National Patient Safety Agency NHS, London. 